\title{
Oral Drug Compounding in Pediatric Patients: a Japanese Perspective
}

\author{
Jumpei Saito ${ }^{1,2 *}$, Miki Akabane ${ }^{1,2}$, Hidefumi Nakamura ${ }^{3}$, Yoichi Ishikawa ${ }^{1}$ and Akimasa Yamatani ${ }^{1,2}$ \\ ${ }^{1}$ Department of Pharmaceuticals, National Center for Child Health and Development, Setagaya-ku, Tokyo, Japan \\ ${ }^{2}$ Division of Clinical Pharmacology and Oral Formulation Development, Department of Clinical Research, National Center for Child Health and \\ Development, Setagaya-ku, Tokyo, Japan \\ ${ }^{3}$ Department of Clinical Research, National Center for Child Health and Development, Tokyo, Japan, Setagaya-ku, Tokyo, Japan
}

${ }^{\star}$ Corresponding author: Jumpei Saito, National Center for Child Health and Development, Okura 2-10-1, Setagaya-ku, Tokyo 157-8535, Japan; Tel: +81 3 3416 0181; Fax: +81-3-3417-5490; Email: saito-jn@ncchd.go.jp

Received: March 27, 2019; Accepted: April 09, 2019; Published: April 12, 2019;

\section{Body Text}

Many medicines used for pediatric patients are not available in pharmaceutical forms adapted to their needs. Actually, most oral medications are developed for adults as tablets and capsules. These forms are lack of dosing flexibility and it does not meet dosage requirements for pediatric patients from neonates to adolescents [1]. According to the updated review that including new pediatric formulations marketed in the United States (US), the country of European Union (EU), and Japan spanning the years 2007 to 2018, 16 kinds of pediatric oral formulations of which 7 drugs are ready-to-use and manipulation is required in 9 drugs, and 51 total new pediatric oral formulations of which 21 drugs are ready-to-use and manipulation is required in 30 drugs [2]. Furthermore in Nigeria, that is one of the lowmiddle income countries, 121 of 143 oral essential medicines (85\%) were not available as flexible solid oral dosage forms and manipulation is forced [3]. When this manipulation is forced, the adult dosage forms are manipulated by either a health care provider, such as pharmacist, or by the parents and caregivers (e.g., crashing or grinding a tablet, capsule opening and sprinkling it into some foods or drink). These processes are called compounding and are commonplace for those medicines that lack pediatric formulations. Compounding procedures are regulated by provincial pharmacy standards, based on guidelines published by the National Pharmacy Regulatory Authorities (NPRA) in Canada or the US Pharmacopeia (USP). However, compounded medicines are not approved by rigorous process in each country such as Food and Drug Administration (FDA), European Medical Agency (EMA), Health Canada, Pharmaceuticals and Medical Devices Agency (PMDA) and the other region's regulatory authorities.

The compounded medication's characteristics and its physical property and specifications are not always known, and its compounding procedure is not well established or controlled and validated before their use in children. This is particularly true with reference to: stability, potency, content uniformity, purity or bioavailability, and so on. First and most important, the administration of the appropriate dose cannot be guaranteed. Moreover, most of the compounded medicines have an unpleasant and bad taste, which leads to adherence challenges. Even if every compounding process is taken to ensure, errors have a potential to do occur. Some compounding is needed in oncologic drugs with the concerns and obvious health risks to health providers and/or caregivers. It has the potential to expose the entire family to these toxic chemical agents. Furthermore, the vehicle (e.g., juice, milk or yogurt) used to dissolve drugs and administer these compounded medicines, or to mask the bad taste of compounded drugs, has a potential to influence drug absorption. Especially, physicians and pharmacist need to be aware of the consequences of compounded medicines for drugs with a narrow therapeutic and safety drug index. Compounding at home also increase the variability in the product by inaccurate measurement, issues with stability or errors in instruction for manipulation [4]. Caregivers often mistake the procedure of the extemporaneous preparation [5]. A lack of bioequivalence study of compounded drugs is also concerned [6]. It is important as the formulation can lead the difference between successful treatment and therapeutic failure.

According to our survey results of 328 hospitals that have a pediatric department in Japan, that account for approximately a half of pediatric department, a total of 320 compounded drugs were identified and most of them were administered as a powder formulation. Top five percentile of compounded drugs were briefly indicated in Table 1. In Canada, the Goodman Pediatric Formulation Center which is a not-for-profit organization that is working as a facilitator between industry, regulatory and reimbursement agencies to bring commercialized pediatric formulations into Canada, also conducted a survey with hospital pharmacists from a dozen pediatric Canadian institutions in 2017. A total of 12 drugs were identified as a priority by at least one third of investigated institutions. Noteworthy, 11 of 16 drugs were same with the result of our survey, suggesting that a lack of appropriate formulation for pediatric patient is common issue in the world and global drug development may be an effective solution (Table 1 , the $5^{\text {th }}$ column).

If the global product development is proceeding, which formulation is acceptable?

In many cases, commercialized pediatric formulations are available in other jurisdictions, such as in the US and in Europe and these are often developed as liquids or suspensions (Table 1, right column). 
Oral liquid medicines have some disadvantages over solid medicines. The major barrier in development of oral liquid formulations is tastemasking of drugs as almost all of health provider for pediatric patients in the US reported that a taste and palatability were the greatest barriers to appropriate medication [7]. The excipients used in the development of a product need to be safe and acceptable for use in children. Excipients are typically used to optimize the formulation of the medicine to improve palatability, shelf-life and/or manufacturing processes [8]. Another problem is that liquid medicines are less chemically stable than solid medicines and require refrigeration in hot climates to guarantee their quality and efficacy. When a company manufactures develop a product for different regions, it may be necessary to adapt tastes and flavors in order to different regional preferences. Having knowledge about caregivers' perceptions would also be needed. These issues become a bottleneck restricting to facilitating the age-appropriate drug development. In 2008, the challenges of ensuring access to appropriate drug formulations for pediatric patients led the World Health Organization (WHO) to propose flexible solid oral dosage forms as the preferred formulations for them [9]. The use of oral solid dosage forms such as dispersible tablets, powders, granules, films or sprinkles for reconstitution have a potential to be an excellent substitute for liquid formulations, because the solid product has typically better stability compared with a liquids. However, the instructions for reconstitution can be complicated for untrained or uneducated individuals, yet it is important that the final product contains the correct dosage for the patients. If these forms are administered in the absence of water they are only applicable to infants who are accepting solid such as a baby food. The risk of any error also remains.

Table 1. The current state of compounding in 328 hospitals that have pediatric departments in Japan and availability in other regulatory authorities.

\begin{tabular}{|c|c|c|c|c|c|}
\hline Active ingredients name & $\begin{array}{c}\text { Frequency of } \\
\text { compounding No. }(\%)\end{array}$ & $\begin{array}{c}\text { Pre-compounded } \\
\text { dosage form (strength) }\end{array}$ & $\begin{array}{l}\text { Compounded } \\
\text { Dosage Form }\end{array}$ & $\begin{array}{c}\text { Common desired flexible } \\
\text { dosage forms among Japan and } \\
\text { Canada }^{\mathrm{a}}\end{array}$ & $\begin{array}{c}\text { Already approved flexible } \\
\text { dosage forms in FDA, MHRA } \\
\text { and EMA }\end{array}$ \\
\hline Dantrolene sodium hydrate & $67(20.6 \%)$ & Capsule (25mg) & Powder & - & N/A \\
\hline Ramelteon & $65(20.0 \%)$ & Tablet (8mg) & Powder & - & N/A \\
\hline Baclofen & $58(17.9 \%)$ & Tablet (10mg) & Powder & $\checkmark$ & Oral liquid $5 \mathrm{mg} / 5 \mathrm{ml}$ \\
\hline Hydrocortisone & $56(17.2 \%)$ & Tablet (10mg) & Powder & $\checkmark$ & N/A \\
\hline Dexamethasone & $49(15.1 \%)$ & Tablet $(0.5 \mathrm{mg})$ & Powder & $\checkmark$ & Elixir $0.1 \mathrm{mg} / \mathrm{mL}$ \\
\hline Prednisolone & $49(15.1 \%)$ & Tablet (5mg) & Powder & $\checkmark$ & Syrup $3 \mathrm{mg} / \mathrm{mL}$ \\
\hline Enalapril maleate & $45(13.9 \%)$ & Powder (5mg) & Powder (dilution) & $\checkmark$ & Oral solution $1 \mathrm{mg} / \mathrm{mL}$ \\
\hline Tadalafil & $42(12.9 \%)$ & Tablet (20mg) & Powder & - & N/A \\
\hline Carvedilol & $41(12.6 \%)$ & Tablet (2.5mg) & Powder & $\checkmark$ & N/A \\
\hline Sildenafil citrate & $30(9.2 \%)$ & Tablet (20mg) & Powder & $\checkmark$ & $\begin{array}{l}\text { Powder for oral suspension } \\
10 \mathrm{mg} / \mathrm{ml}\end{array}$ \\
\hline Clonidine hydrochloride & $30(9.2 \%)$ & Tablet $(75 \mu \mathrm{g})$ & Powder & $\checkmark$ & N/A \\
\hline Levothyroxine sodium & $30(9.2 \%)$ & Tablet $(50 \mu \mathrm{g})$ & Powder & $\checkmark$ & Oral solution $100 \mu \mathrm{g} / 5 \mathrm{ml}$ \\
\hline Diazoxide & $26(8.0 \%)$ & Tablet (25mg) & Powder & $\checkmark$ & Oral suspension $50 \mathrm{mg} / \mathrm{mL}$ \\
\hline Aspirin & $23(7.1 \%)$ & Powder (1g/g) & Powder (dilution) & - & N/A \\
\hline Propranolol hydrochloride & $22(6.8 \%)$ & Tablet (10mg) & Powder & $\checkmark$ & Oral solution $8 \mathrm{mg} / \mathrm{mL}$ \\
\hline Methotrexate & $22(6.8 \%)$ & Tablet $(2.5 \mathrm{mg})$ & Powder & - & Oral solution $2 \mathrm{mg} / \mathrm{ml}$ \\
\hline
\end{tabular}

a. The information about compounding in Canadian hospital was provided by the Goodman Pediatric Formulations Centre. Check marks $(\checkmark)$ indicate the common drugs that are desired from both Canada and Japan.

b. Approved drug information was searched using the websites provided from Food and Drug Administration (FDA) in the United States, Medicines and Healthcare products Regulatory Agency (MHRA) in the United Kingdom, and European Medical Agency (EMA)

Compounded drugs in the top 5 percentile was indicated. No., number; N/A, not available.

From these compelling issues, the Academy of Pharmaceutical Science and Technology in Japan and its subcommittees named 'the individualized medicine focus group' and 'the clinical formulation focus group', decided to prepare countermeasures in medically ensured compounding procedures. We aimed to collect accurate information about the present status of compounding and unclose what information is needed to the medically ensured compounding procedure. 
To facilitate the drug development globally, no regulatory and financial drivers to develop age-appropriate medicines for pediatric patient become a heavy drag, especially about off-patent drugs. In the European countries, there is a significant number of existing drugs where age-appropriate formulations are needed $[10,11]$. Almost all of these drugs are generic drugs developed in the remote past for which there are no incentives or any intellectual property protection, making these drugs less interesting to invest time and cost. Requesting an age-appropriate drug development to industrial companies is one of the best solutions, however, ensuring the quality of compounded formulation by the health professionals is required as an urgent issue. While the future of ideal pediatric oral formulations may increasingly be with taste-masked, preservative-free, and user-friendly formulations including multi-particulate solid dosage forms such as mini-tablets, orally disintegrating tablets, and granules, to assure the quality of compounded drug that is not on the radar of manufacturer is needed.

\section{Acknowledgement}

This work was supported by a Research Program from the Japanese Agency for Medical Research and Development under Grant Number JP19mk0101134, awarded to H.N. We are grateful to collaborated 328 hospital pharmacists who supported us to perform our survey.

\section{References}

1. Venables R, Marriott J, Stirling H (2012) FIND OUT: key problems with children's medicines formulations ... It's a taste issue! Int J Pharm Pract 20: 23.

2. Strickley RG (2019) Pediatric oral formulations: an updated review of commercially available pediatric oral formulations since 2007. J Pharm Sci 108: 1335-1365.

3. Orubu ES, Tuleu C (2017) Medicines for children: flexible solid oral formulations. Bull World Health Organ 95: 238-240.

4. Richey RH, Shah UU, Peak M, Craig JV, Ford JL, et al. (2013) Manipulation of drugs to achieve the required dose is intrinisic to paediatric practice but is not supported by guidelines or evidence. BMC Pediatr 13: 1-8.

5. Tomlin S, Cokerill H, Costello I, Griffith R, Hicks R, et al. (2009) Making medicines safer for children - guidance on the use of unlicensed medicines in paediatric patients. Guidelines 1-12.

6. Batchelor HK, Marriott JF (2015) Formulations for children: problems and solutions. Br J Clin Pharmacol 79: 405-418.

7. Milne CP, Bruss JB. (2008) The economics of pediatric formulation development for off-patent drugs. Clin Ther 30: 2133-2145.

8. Fabiano V, Mameli C, Zuccotti GV. (2011) Paediatric pharmacology: remember the excipients. Pharmacol Res 63: 362-365.

9. WHO. Annex 5. (2012) Development of paediatric medicines: points to consider in formulation. World Health Organ Tech Rep Ser 970: 197-225.

10. EMA. (2013) Revised provisional priority list for studies into off-patent paediatric medicinal products. EMA/98717/2012: 1-12.

11. WHO. (2015) WHO Model List of Essential Medicines for Children. $5^{\text {th }}$ Edition: $1-42$.

Citation:

Saito J, Akabane M, Nakamura H, Ishikawa Y, Yamatani A (2019) Oral Drug Compounding in Pediatric Patients: a Japanese Perspective. J Pharmacol Pharm Res Volume 2 (3): 1-3. 